\title{
Distributed Attention for Grounded Image Captioning
}

\author{
Nenglun Chen \\ The University of Hong Kong \\ Youtu Lab, Tencent \\ Lei Yang \\ The University of Hong Kong \\ Haolei Yuan \\ Youtu Lab, Tencent
}

\author{
Xingjia Pan \\ Youtu Lab, Tencent
}

Zhiwen Lin
Youtu Lab, Tencent

Xiaowei Guo

Youtu Lab, Tencent

\author{
Runnan Chen \\ The University of Hong Kong
}

Yuqiang Ren
Youtu Lab, Tencent

Feiyue Huang

Youtu Lab, Tencent

\author{
Wenping Wang \\ The University of Hong Kong
}

\begin{abstract}
We study the problem of weakly supervised grounded image captioning. That is, given an image, the goal is to automatically generate a sentence describing the context of the image with each noun word grounded to the corresponding region in the image. This task is challenging due to the lack of explicit fine-grained region word alignments as supervision. Previous weakly supervised methods mainly explore various kinds of regularization schemes to improve attention accuracy. However, their performances are still far from the fully supervised ones. One main issue that has been ignored is that the attention for generating visually groundable words may only focus on the most discriminate parts and can not cover the whole object. To this end, we propose a simple yet effective method to alleviate the issue, termed as partial grounding problem in our paper. Specifically, we design a distributed attention mechanism to enforce the network to aggregate information from multiple spatially different regions with consistent semantics while generating the words. Therefore, the union of the focused region proposals should form a visual region that encloses the object of interest completely. Extensive experiments have demonstrated the superiority of our proposed method compared with the state-of-the-arts.
\end{abstract}

\section{CCS CONCEPTS}

- Computing methodologies $\rightarrow$ Natural language generation; Object detection.

\section{KEYWORDS}

Image captioning; Visual grounding; Weakly supervised learning; Neural networks

Permission to make digital or hard copies of all or part of this work for personal or classroom use is granted without fee provided that copies are not made or distributed for profit or commercial advantage and that copies bear this notice and the full citation on the first page. Copyrights for components of this work owned by others than ACM must be honored. Abstracting with credit is permitted. To copy otherwise, or republish, to post on servers or to redistribute to lists, requires prior specific permission and/or a fee. Request permissions from permissions@acm.org.

MM '21, October 20-24, 2021, Virtual Event, China

(c) 2021 Association for Computing Machinery.

ACM ISBN 978-1-4503-8651-7/21/10 ..\$15.00

https://doi.org/10.1145/3474085.3475354
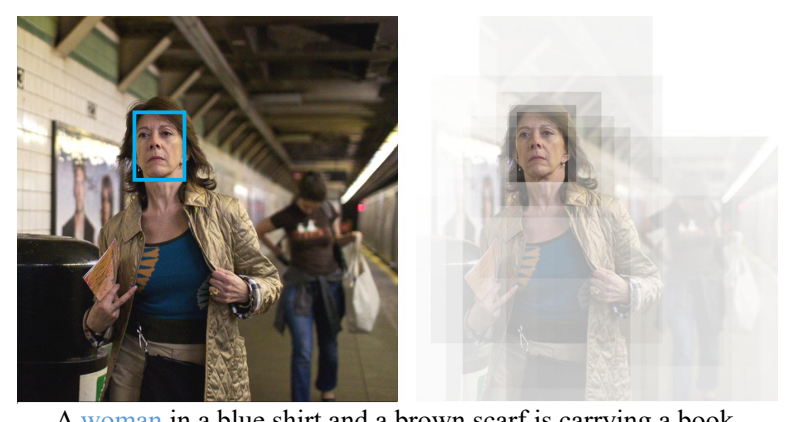

A woman in a blue shirt and a brown scarf is carrying a book.

Figure 1: The partial grounding problem. The captions and the corresponding attention were generated with the baseline method. The attended region of the word 'woman' only covers the 'face'.

\section{ACM Reference Format:}

Nenglun Chen, Xingjia Pan, Runnan Chen, Lei Yang, Zhiwen Lin, Yuqiang Ren, Haolei Yuan, Xiaowei Guo, Feiyue Huang, and Wenping Wang. 2021. Distributed Attention for Grounded Image Captioning. In Proceedings of the 29th ACM International Conference on Multimedia (MM '21), October 20-24, 2021, Virtual Event, China. ACM, New York, NY, USA, 10 pages. https://doi.org/10.1145/3474085.3475354

\section{INTRODUCTION}

Describing a given scene is considered an essential ability for humans to perceive the world. It is thus important to develop intelligent agents with such an ability, formally known as image captioning that generates the natural language descriptions based on the given images $[9,15,23,46]$. A further pursuit in the image captioning task has emerged: not only the captions should semantically reflect the content of the image, but also the words in the generated captions should be grounded in the image, i.e., finding visual regions in the image that corresponds to the words. This way, more interpretable captions are expected to be generated.

To this end, the mainstream works leverage attention mechanisms that focus on certain regions of images to generate more grounded captions. Pioneering works $[25,52,59]$ have explored using annotated bounding box as attention supervision for each 
visually groundable word or noun phrase in the training stage, and led to desirable improvement on the interpretability of the generated captions. However, acquiring such region-word alignment annotations is expensive and time-consuming.

Recently, several attempts have been made to generate more grounded attention with weak supervision $[26,31,60]$. These methods mainly focus on designing various regularization schemes to produce attended regions to enclose the visual entities corresponding to the words in the generated captions. Despite the promising results achieved by the weakly supervised methods, their performances are still far from the fully supervised baselines. One main issue is that the attention for generating the visually groundable word without ground-truth grounding supervision may only focus on the most discriminant regions, failing to cover the entire object. See Fig. 1 for example, the grounded region of the word 'woman' generated by our baseline network only covers the facial part. While such grounding region identify the correct person ('the woman in blue shirt'), the network largely ignores the body parts presented in the image, which means that it fails to visually distinguish the word 'woman' and the word 'face'. This problem is important, especially when the input images are not accessible to the users (e.g. voice assist system for blind person). In such cases, whether grounding on partial or the whole body may affect some follow-up decisions.

Such a problem, termed as the partial grounding problem in our paper, is usually seen in the grounded image captioning task with weak supervision. This is primarily attributed to the fact that the objects of interest in the images are usually partially covered by many redundant region proposals, and choosing the most salient proposal inevitably leads to the described problem. In Fig. 2, we statistically analyze the grounding error of the baseline architecture on Flickr 30k Entities [36] dataset by casting it into three categories including partial grounding, enlarged grounding, and deviated grounding. As we can see that, the partial grounding problem contributes to a large portion of the grounding error. This further confirms the necessity to solve the partial grounding problem.

Based on the observation, we propose a simple yet effective method to alleviate the partial grounding problem. The ingredient of our method is a distributed attention mechanism that enforces the network to attend to multiple semantically consistent regions. In this way, the union of the attended regions should form a visual region that encloses the object completely. We follow the previous method [59] and build our baseline network based on the widely used up-down architecture [2]. The attention module in the baseline network is augmented with multiple branches, and region proposal elimination is used to enforce different attention branches to focus on different regions. We conduct various experiments to verify the effectiveness of our method. From our experiments, we illustrate that the partial grounding problem is a crucial issue that produces inferior grounding results, and adding our distributed attention to the baseline method can effectively achieve a large performance gain on grounding accuracy than those produced by the state-ofthe-art methods relying on sophisticated network designs.

In summary, our technical contributions are summarized:

- We find that alleviating the partial grounding issue is critical to promote grounding performance, which is ignored in all previous methods.

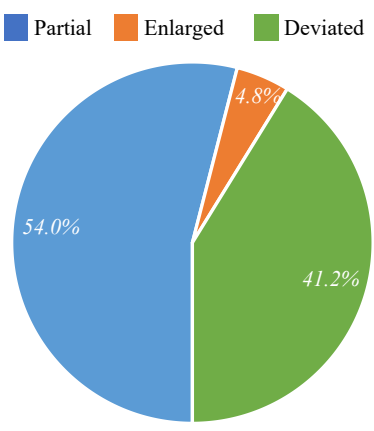

(a) Statistics

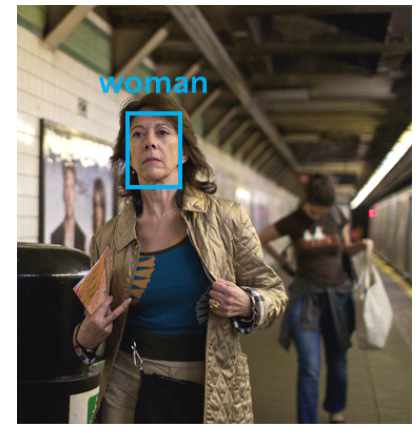

(c) Partial Grounding

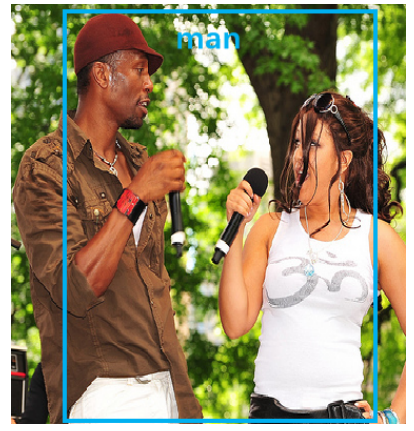

(b) Enlarged Grounding

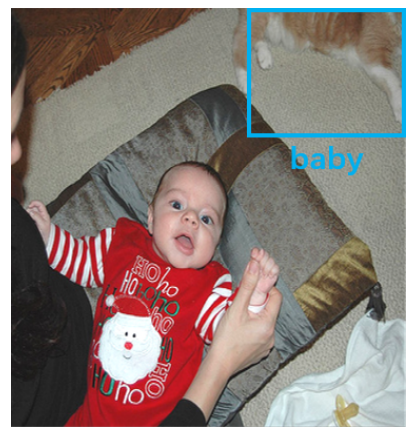

(d) Deviated Grounding
Figure 2: Error analysis of the baseline method.

- We propose a novel solution to the partial grounding issue by introducing a distributed attention mechanism that enforces the network to aggregate the semantically consistent regions across the entire image.

- By testing our method on the Flickr30k Entities dataset and ActivityNet Entities dataset, we achieve significant gain on grounding accuracy compared with state-of-the-art methods.

\section{RELATED WORK}

Grounded Image Captioning. Image captioning is an important task at the juncture of computer vision and natural language processing. Traditional methods [10, 23, 32, 54] mainly adopts the pre-defined templates with slots that are filled with visual cues detected on the images. The performance of these methods is limited. With the advent of deep neural networks, the caption quality has been greatly improved. State-of-the-art methods $[2,9,15,18$, $30,49,50]$ make use of various form of the attention mechanism for attending to different regions of the images while generating captions. The attention mechanism can be applied to various types of data including spatial grids [30], semantic metadata $[49,50]$ as well as object region proposals $[2,18]$. Despite the great success achieved in terms of captioning performance of these methods, the grounding accuracy is still not satisfactory.

Several attempts have been made for improving the grounding accuracy. Some pioneering works $[25,52,54,59]$ obtained improved grounding results with the help of the attention mechanism in a 
fully supervised manner. However, labeling the word-region correspondences is time-consuming and difficult to scale up to large datasets. Recently, researchers started to use different kinds of regularization schemes to improve the grounding accuracy in a weakly supervised manner. Zhou et al. [60] adopted the teacher-student scheme and distilled the knowledge learned by a Part-of-Speech enhanced image-text matching network [24] to improve the grounding accuracy of the image captioning model. Ma et al. [31] employed the cyclical training regimen as a regularization, in which a generator and a localizer are trained jointly to regularize the attention. Liu et al. [26] proposed the Prophet Attention, which uses future information to compute the ideal attention and then takes the ideal attention as regularization to alleviate the deviated attention. The abovementioned methods utilized different regularization schemes to improve the grounding accuracy, however, they all neglect the partial grounding issue as described previously. To this end, we propose a distributed attention mechanism for aggregating semantically consistent regions to alleviate the partial grounding problem.

Visual Grounding. The visual grounding task aims at learning fine-grained correspondences between image regions and visually groundable noun phrases. Existing works can be roughly divided into two categories: supervised and weakly supervised. The supervised methods [12, 29, 35, 51] took bounding boxes as supervision to enforce the alignment between image regions and noun phrases, and have achieved remarkable success. However, annotating the region word alignments is expensive and time-consuming, which makes it difficult to scale to large datasets.

Weakly supervised methods [6, 16, 27, 28, 39, 47] aimed at learning the correspondence with only image-caption pairs. Some studies utilize reconstruction regularization [27, 39] to learn visual grounding. Rohrbach et al. [39] first generate a candidate bounding box by the attention mechanism and then reconstruct the phrase based on the selected bounding box. Liu et al. [27] further boost the grounding accuracy by introducing the contextual entity and model the relationship between the target entity (subject) and contextual entity (object). Contrastive learning is also exploited in several method [16, 47]. Wang et al. [47] learn a score function between the region-phrase pairs for distilling knowledge from a generic object detector for weakly supervised grounding. Gupta et al. [16] maximize a lower bound on mutual information between sets of the region features extracted from an image and contextualized word representations. Chen et al. [6] facilitate weakly supervised grounding by considering both visual and language consistency and leveraging complementary knowledge from the feature extractor. Our task is different from visual grounding in that our caption is to be generated rather than given for grounding.

Weakly Supervised Object Detection. WSOD aims to learn the localization of objects with only image-level labels (e.g., ones given for classification) and can be roughly divided into methods based on region proposals or class activation maps (CAMs) [58] Proposal-based approaches $[4,14,42]$ formulate this task as multiple instance learning. Bilen et al. [5] select proposals by parallel detection and classification branches in deep convolutional networks. Contextual information [19], attention mechanism [43], gradient map [40] and semantic segmentation [48] are leveraged to learn accurate object proposals. CAM-based methods [33, 41, 41, 55, 57] produce localization maps by aggregating deep feature maps using a class-specific fully connected layer. Despite the simplicity and effectiveness of CAM-based methods, they suffer from identifying small discriminative parts of objects. To improve the activation of CAMs, several methods $[8,41,53,55]$ adopted adversarial erasing on input images or feature maps to drive localization models focusing on extended object parts. SPG [56] and $\mathrm{I}^{2} \mathrm{C}$ [57] increased the quality of localization maps by introducing the constraint of pixel-level correlations into the network. SPA [33] and TS-CAM [13] obtain accurate localization maps with the help of long-range structural information. Different from the WSOD task, our task leverages the correctly attended regions for caption generation.

\section{METHODOLOGY}

In this section, we first give the notations of image captioning (Sec 3.1). We then briefly introduce the attention based encoderdecoder baseline network as our image captioning baseline ( $\operatorname{Sec} 3.2$ ). In Sec 3.3, we describe in detail the proposed distributed attention mechanism for aggregating semantically consistent partial regions for more grounded image captioning.

\subsection{Notations}

The generic image captioning task aims at generating a sentence $Y$ to describe the context of a given image $I$. We follow the pioneering works $[31,59]$ on weakly supervised image captioning and present the input image as a set of region proposals $R=\left\{r_{1}, r_{2}, \ldots, r_{N}\right\}\left(r_{i} \in\right.$ $\mathbb{R}^{d}$ ) and the image feature map $f_{c}$, where $N$ and $d$ are the number and the feature dimension of the region proposals, respectively. We generate $r_{i}$ with Faster RCNN [37] while $f_{c}$ is extracted from a pretrained ResNet [17] model. The generated sentence is represented as a sequence of one-hot vectors $Y=\left\{y_{1}, y_{2}, \ldots, y_{T}\right\}\left(y_{t} \in \mathbb{R}^{s}\right)$, where $T$ is the length of the sequence and $s$ is the size of the vocabulary.

\subsection{Revisit the Baseline Method}

Our method is built on GVD [59] except for the self-attention loss for region feature embedding. Fig. 3(b) shows the core part of the GVD, named language generating module, which is an extension of the widely used Bottom-up and top-down attention network [2] for the image captioning task. Specifically, the language generating model consists of two LSTM layers, the attention LSTM layer, and the language LSTM layer. The attention LSTM is used to identify the importance of different regions in the input image for generating the next word. It takes as input the global image feature vector $v_{g}$, the previous word embedding vector $e_{t-1}=W_{e} y_{t-1}$ and the previous hidden state $h_{t-1}^{1}$, and encode them into current hidden state $h_{t}^{1}$ :

$$
h_{t}^{1}=\operatorname{LSTM}_{1}\left(\left[v_{g} ; e_{t-1}\right], h_{t-1}^{1}\right),
$$

where $W_{e}$ is the learnable word embedding matrix, $[\because ; \cdot]$ denotes the concatenation operation and we obtain $v_{g}$ by applying global average pooling on the image feature map $f_{c}$. Note that, the description of the cell states for both LSTM layers is omitted for notational clarity.

To obtain the attention weight $\alpha_{t}$ over the image regions, the hidden state $h_{t}^{1}$ produced by the above-mentioned attention LSTM layer is further fed into an attention module followed by a softmax 


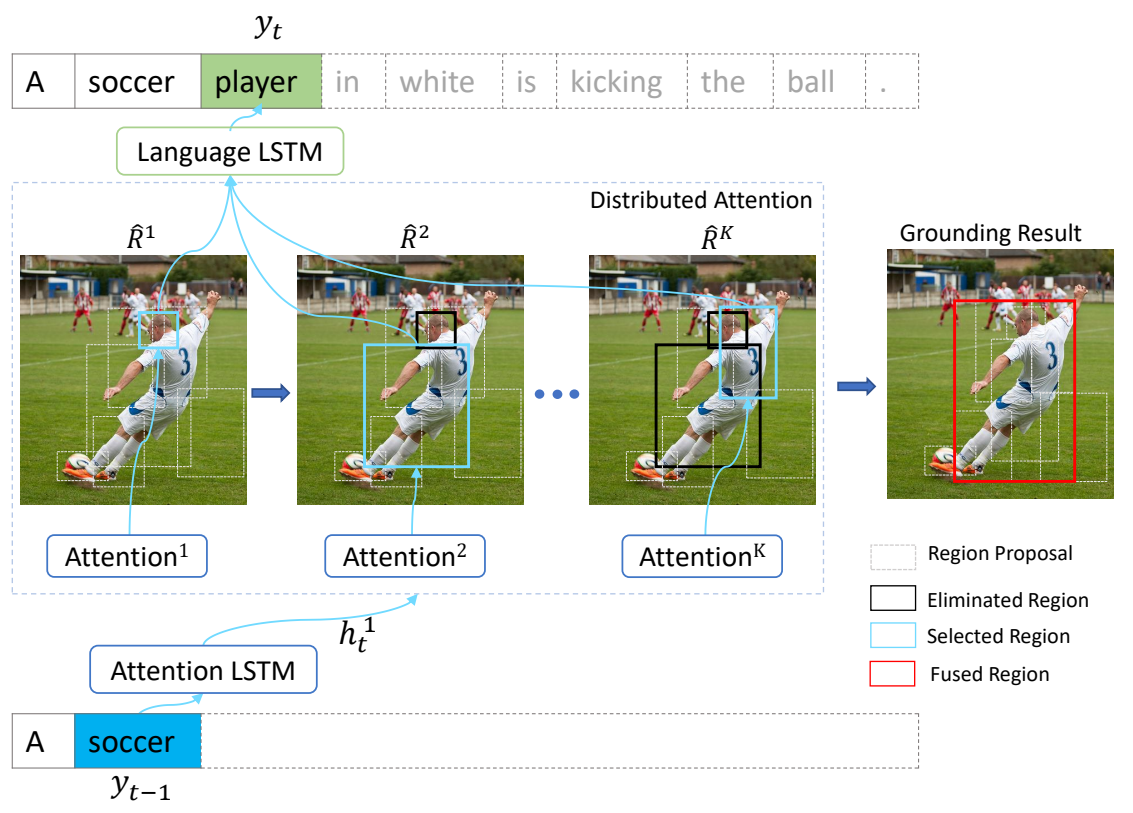

(a)

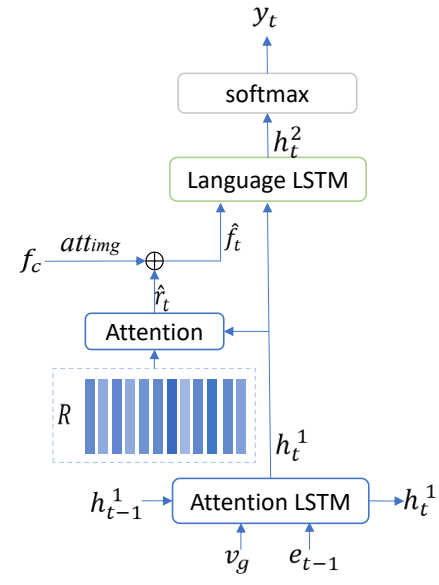

$\bigoplus$ Element wise addition

Figure 3: The pipeline of our proposed method (a), and the detailed framework of the baseline network (b). The attention branches in the distributed attention module are applied iteratively to discover semantically consistent regions. Note that, we do not show all the region proposals for a clear illustration.

layer as:

$$
\begin{aligned}
z_{i, t} & =W_{a} \tanh \left(W_{r} r_{i} \oplus W_{h} h_{t}^{1}\right), \\
\alpha_{t} & =\operatorname{softmax}\left(z_{t}\right),
\end{aligned}
$$

where $W_{a}, W_{r}$ and $W_{h}$ are the learnable weight matrices and $\oplus$ is the element-wise addition operation. Given the attention weight $\alpha_{t}$, the attended region feature $\hat{r}_{t}$ can be calculated as the weighted combination of the regions features:

$$
\hat{r}_{t}=\alpha_{t}^{\top} R
$$

where $R \in \mathbb{R}^{N \times d}$ is a feature matrix whose column represents the feature of a region proposal. Following [31, 59], we also enhance the attended region feature $\hat{r}_{t}$ with the image features $f_{c}$ to get the attended image feature $\hat{f}_{t}$ :

$$
\hat{f}_{t}=\hat{r}_{t}+\operatorname{att}_{\text {img }}\left(f_{c}\right)
$$

where $a t t_{i m g}$ is the attention block for aggregating the image feature map $f_{c}$ into a feature vector.

Finally, the attended image feature $\hat{f}_{t}$ is fed into the language LSTM for producing the conditional probability distribution $p\left(y_{t} \mid y_{1: t-1}\right)$ of the next word $y_{t}$ over all possible outputs as:

$$
\begin{array}{r}
h_{t}^{2}=\operatorname{LSTM}_{2}\left(\left[h_{t}^{1}, \hat{f}_{t}\right], h_{t-1}^{2}\right), \\
p\left(y_{t} \mid y_{1: t-1}\right)=\operatorname{softmax}\left(W_{o} h_{t}^{2}\right),
\end{array}
$$

where $y_{1: t-1}$ denotes the sequence of the previously predicted words, $p\left(y_{t} \mid y_{1: t-1}\right)$ denotes the conditional probability of the word $y_{t}$ given the previous words, $h_{t}^{2}$ is the hidden state of the language LSTM and $W_{o}$ is the learnable weight.

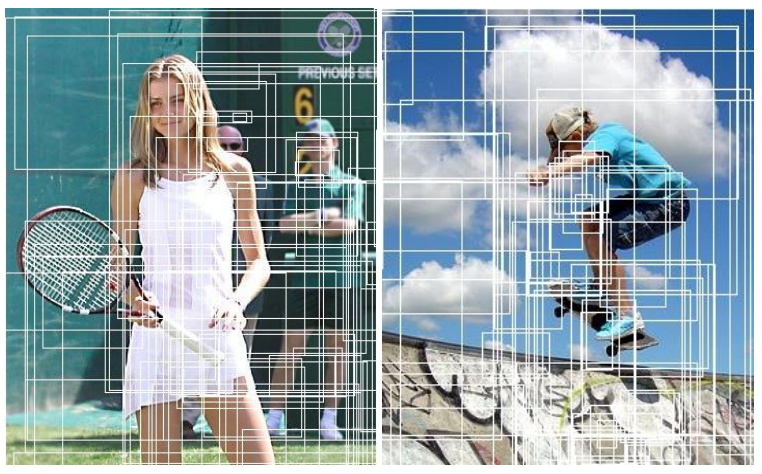

Figure 4: The extracted region proposals (white bounding boxes) are redundant and the objects are partially covered by many region proposals.

In the training phase, the network is optimized by adopting the teacher forcing strategy given the ground truth caption sequence $Y^{*}=\left\{y_{1}^{*}, y_{2}^{*}, \ldots, y_{T}^{*}\right\}$. The training objective is to minimize the following cross-entropy loss $L_{C E}(\theta)$ :

$$
L_{C E}(\theta)=-\sum_{t=1}^{T} \log \left(p_{\theta}\left(y_{t}^{*} \mid y_{1: t-1}^{*}\right)\right)
$$

where $\theta$ denotes the trainable parameters. The region with the maximum attention weight will be selected as the grounding result. 


\subsection{Region Aggregation with Distributed Attention}

In this section, we introduce our distributed attention mechanism for aggregating semantically consistent regions to resolve the partial grounding problem in detail.

As shown in Fig. 4, the extracted region proposals by a pretrained Faster-RCNN are redundant with each object partially covered by many region proposals. It is likely that the attention mechanism used in the baseline network will only identify parts of the objects which are the most discriminative.

Based on the above discussion, we propose the distributed attention mechanism for aggregating semantically consistent regions for generating the words. Fig. 3(a) illustrates the overall pipeline of our proposed method. Specifically, to make the attention focus on different regions, we augment the attention module in the baseline network with $K$ attention branches, and enforce the focused regions of all the attention branches to be mutually exclusive by iteratively applying the attention branches on the region proposals:

$$
\begin{aligned}
& a_{t}^{k}=\operatorname{Attention}^{k}\left(\hat{R}^{k}, h_{t-1}^{1}\right), \\
& \hat{R}^{k}=\left\{r_{i} \in R, r_{i} \notin M^{k-1}\right\}
\end{aligned}
$$

where Attention $^{k}()$ is the function for computing the attention weights with $k$-th attention branch over the region proposals as described in Equ. 2, $k \in[1, K]$ is the branch index, $\hat{R}^{k}$ denotes the set of region proposals used in the attention branch $k, a_{t}^{k}$ is the attention weights predicted by the $k$-th attention branch and $M^{k-1}$ denotes $k-1$ region proposals selected by previous $k-1$ attention branches. Note that these attention branches do not share the same weights. The attended features of all $K$ attention branches are further passed through language LSTM (Equ. 3, 4, 5) to get $K$ outputs.

At the training stage, we use the teacher-forcing strategy and apply the cross-entropy loss to all the $K$ outputs. During testing, at each time step, we select the word predicted by the most number of attention branches and combine the corresponding regions together into a single region and use it as the grounding output.

Through our method, the distributed attention model will learn to aggregate semantically consistent regions when generating the word to alleviate the partial grounding problem. The union of the attended regions should form a visual region that encloses the object of interest completely.

\section{EXPERIMENTS}

\subsection{Experimental Settings}

Datasets. We conducted the main experiments on the widely used Flickr30k-Entities dataset [36]. It contains $31 k$ images in total, and each image is annotated with 5 sentences. In addition, it also contains $275 k$ bounding box annotations and each bounding box corresponds to a visually groundable noun phrase in the caption. Following GVD [59], we used the data split setting from Karpathy et al. [20], which has $29 k$ images for training, $1 k$ images for validation, and $1 k$ for testing. The vocabulary size of the dataset is 8639 .

Evaluation Metrics. We used the standard captioning evaluation toolkit [7] to measure the captioning quality. Four commonly used
Table 1: Comparison with other state-of-the-art methods on the Flickr30k Entities test set.

\begin{tabular}{lccccccc}
\hline Method & \multicolumn{3}{c}{ Captioning Eval } & \multicolumn{3}{c}{ Grounding Eval } \\
\cline { 2 - 8 } & B@1 & B@4 & M & C & S & $F 1_{\text {all }}$ & $F 1_{\text {loc }}$ \\
\hline Supervised methods & & & & & & & \\
Cyclical[31] & 69.0 & 26.8 & 22.4 & 61.1 & 16.8 & 8.44 & 22.78 \\
GVD[59] & 69.9 & 27.3 & 22.5 & 62.3 & 16.5 & 7.55 & 22.2 \\
\hline Weakly supervised methods & & & & & & \\
GVD[59] & 69.2 & 26.9 & 22.1 & 60.1 & 16.1 & 3.88 & 11.70 \\
Prophet[26] & - & 27.2 & 22.3 & 60.8 & 16.3 & 5.45 & 15.30 \\
Zhou et al.[60] & $\mathbf{7 1 . 4}$ & $\mathbf{2 8 . 0}$ & $\mathbf{2 2 . 6}$ & $\mathbf{6 6 . 2}$ & $\mathbf{1 7 . 0}$ & 6.53 & 15.79 \\
Cyclical[31] & 69.9 & 27.4 & 22.3 & 61.4 & 16.6 & 4.98 & 13.53 \\
\hline Ours & 69.2 & 27.2 & 22.5 & 62.5 & 16.5 & $\mathbf{7 . 9 1}$ & $\mathbf{2 1 . 5 4}$ \\
\hline
\end{tabular}

language evaluation metrics are reported, i.e., BLEU [34], METEOR [11], CIDEr [45] and SPICE [1].

To evaluate the grounding performance, we used the metrics $F 1_{\text {all }}$ and $F 1_{\text {loc }}$ defined in GVD [59]. For $F 1_{\text {all }}$, a prediction is considered as correct if the object word is correctly generated and the Intersection-over-Union (IOU) of the predicted bounding box and the ground truth bounding box is larger than 0.5 . In $F 1_{l o c}$, only the correctly generated words are considered. Both metrics were averaged over classes.

Implementation Details. We implemented our method with PyTorch, and all the experiments were conducted on two V100 GPUs. Following the previous works [26, 31, 59], we adopted the widely used Faster R-CNN network [37] pre-trained on Visual Genomes [22] by GVD [59] to extract 100 region proposals for each image. The word embedding dimension in the captions was set to 512, and the word embedding layer was trained from scratch. The dimension of the hidden states for both Attention and Language LSTM was set to 1024 .

The network was optimized with Adam Optimizer [21], with an initial learning rate set to $5 e^{-4}$ and decayed by a factor of 0.8 for every three epochs. The batch size was set to 64 . We trained the network with a single attention branch for 20 epoches and included the other branches in the training loop afterward. The whole training process takes less than one day.

\subsection{Comparison with Prior Work}

Quantitative Comparison. We compared our method with stateof-the-art weakly supervised grounded image captioning methods, including GVD [59], Prophet [26], Zhou et al. [60] and Cyclical [31], to see the effectiveness of our method on the grounding accuracy. Note that, in Zhou et al. [60], various techniques are used to improve the captioning performance including SCST [38] and scheduled sampling [3], while we only employ the standard cross-entropy loss used in the baseline network during training.

As shown in Table. 1, by applying our proposed distributed attention module on the baseline method, we achieved significant improvement on the grounding accuracy $\left(F 1_{\text {all }}\right.$ and $\left.F 1_{\text {loc }}\right)$ compared with other weakly supervised grounded image captioning methods. By comparing our method with the supervised baseline method in Cyclical, the grounding performance of our method only inferiors a little. It further confirmed the necessity of solving the partial grounding problem in this task. 

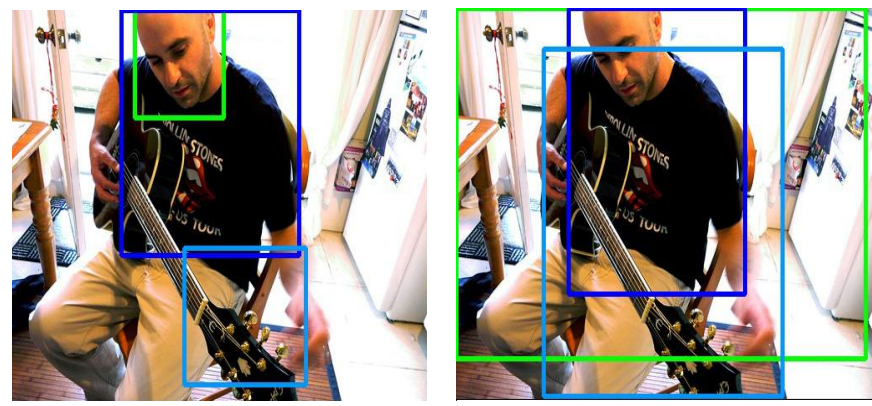

Baseline: A man in a black shirt plays a guitar. Ours: A man in a black shirt plays a guitar.

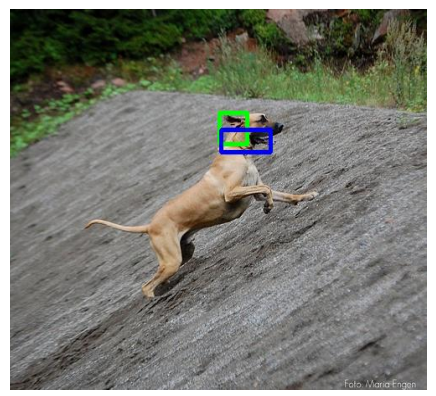

Baseline: $\underline{A}$ dog is jumping up to catch a large rock.

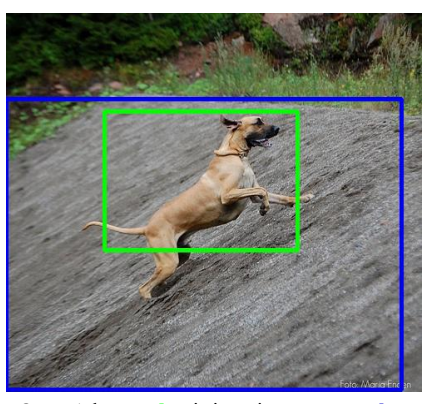

Ours: A brown dog is jumping over a rock.

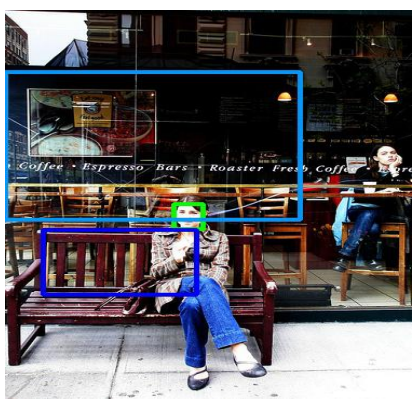

Baseline: A woman sitting on a bench in front of a store.

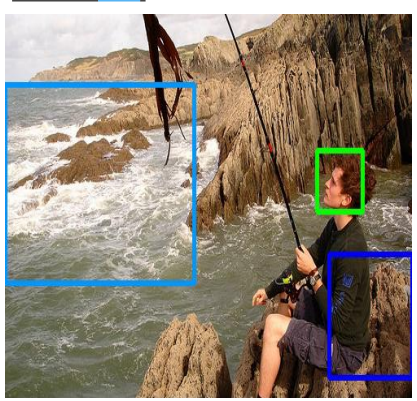

Baseline: A man sitting on a rock in the water.

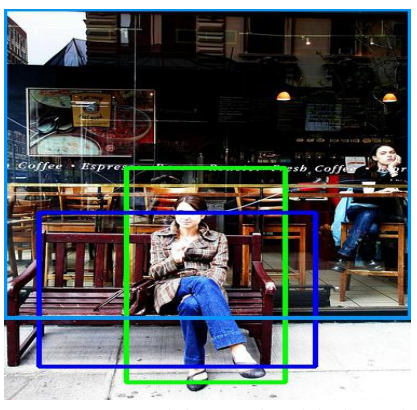

Ours: A woman sitting on a bench in front of a store.

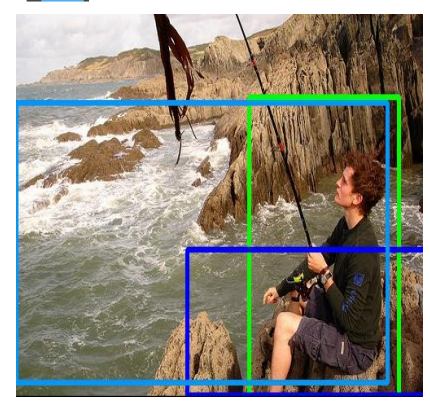

Baseline: A man sitting on a rock in the water.

Figure 5: Results of the generated captions and the grounding regions by comparing our method with the baseline.

Qualitative Comparison. We qualitatively compare our method with the baseline method to see how the partial grounding problem can be alleviated. As shown in Fig. 5, the predicted grounding regions of our method are more accurate and can cover the whole objects, while the selected regions of the baseline method may only cover the most salient parts.

Performance on ActivityNet-Entities. We further tested the performance of our method on the ActivityNet-Entities dataset [59] to see if our method works or not on the grounded video description task. By extending GVD with our distributed attention module, we achieved significant improvement on the validation set for both $F 1_{\text {all }}$ (from 3.70 to 5.32) and $F 1_{l o c}$ (from 12.70 to 21.06) with the captioning performance keeping comparable. This further confirmed the effectiveness of our proposed method.

\subsection{Qualitative Results.}

Example Results. In Fig. 6, we present some example results including the generated captions as well as the corresponding grounding results. As we can see that, our method is able to generate fine grained image captions with accurate groundings for both foreground objects (e.g., 'man') and the background (e.g., 'beach').

Visualization of the Distributed Attention. We visualize the distributed attention in Fig. 7 to see how the region proposals are selected and fused. When generating a word, the distributed attention will attend to multiple semantically consistent regions with different locations, and the attended region proposals with the same semantic meanings will be fused to get the grounding results. We can see that our distributed attention has the ability to aggregate semantically consistent regions to enclose the object of interest completely and thus alleviate the partial grounding problem.

\subsection{Ablation Study}

Effect of $K$. We tested the performance of our method with a varied number of attention branches $K$. As shown in Table 2, the grounding performance of our method outperformed the state-of-the-art methods by a large margin when the number of attention branches $K$ is larger than 3 . When $K$ is smaller than 3 , the grounding accuracy is degraded. The main reason is that, due to the redundant region proposals extracted with Faster-RCNN, the distributed attention module is not able to discover enough regions when $K$ is too small. Effect of Region Proposal Elimination. As shown in Table 3 we tested the performance of our method without region proposal elimination. Our method can also improve the grounding accuracy without region proposal elimination. That's mainly because, different initialization of the attention branches will lead to slightly different attention results, and the partial grounding problem can also be alleviated. The use of the region proposal elimination is to explicitly enforce the distributed attention module to focus on different semantically consistent regions to further boost the grounding performance.

Relationship with Multi-head Attention. Our method has a close relationship with multi-head attention proposed in [44]. Here, we compared our distributed attention with multi-head attention by replacing the attention module in our baseline network with multihead attention and tested it by varying the number of attention heads from 2 to 6 . The best performing result is achieved with the number of attention head setting to 4 , that is 4.75 for $F 1_{\text {all }}$ and 


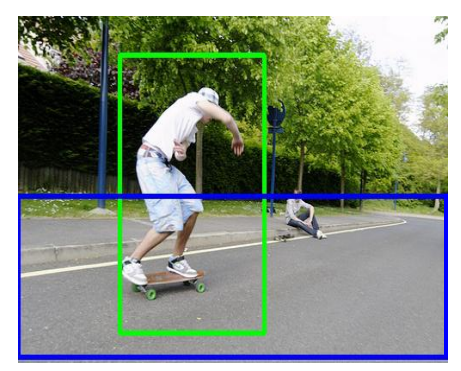

A skateboarder is skateboarding down the street.

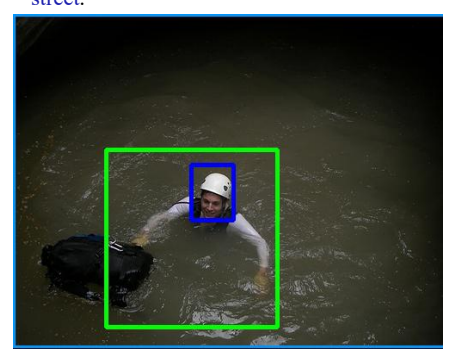

A man in a white helmet is in the water.

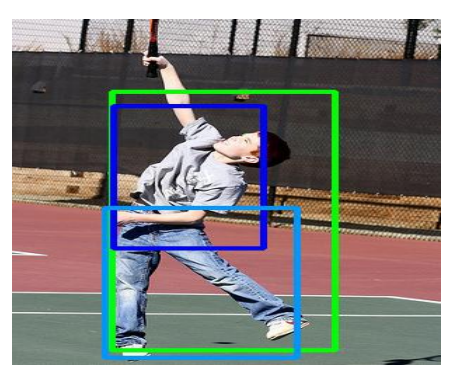

A young boy in gray shirt and blue jeans is playing with tennis.

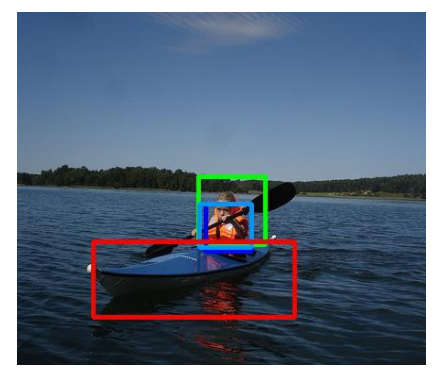

A boy in an orange life vest is paddling a blue boat.

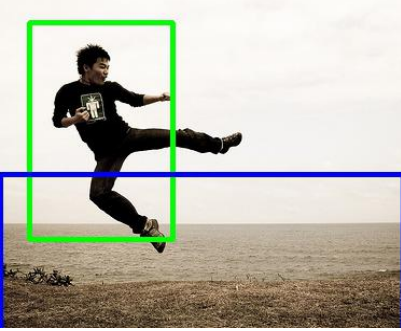

A man in black is jumping in the air on the beach.

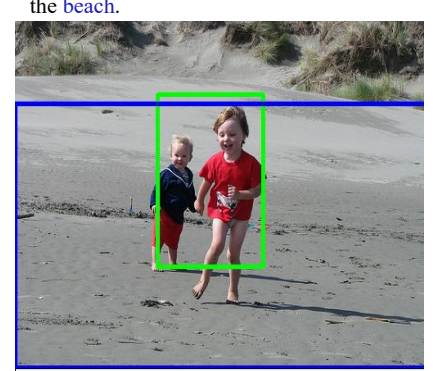

Two children are walking on the beach.

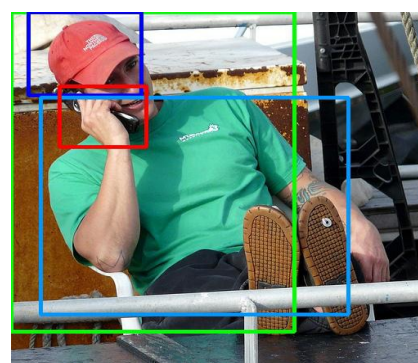

A man in a red hat and green shirt is talking on a cellphone.

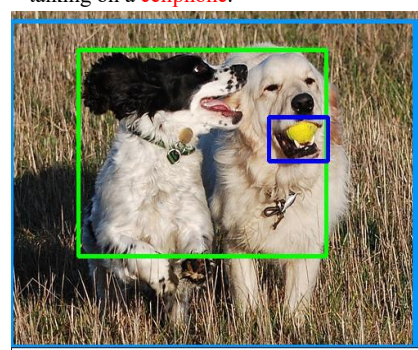

Two dogs are playing with tennis ball in a field.

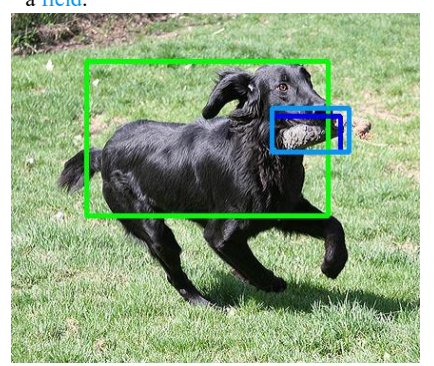

A dog is running with a stick in its mouth.

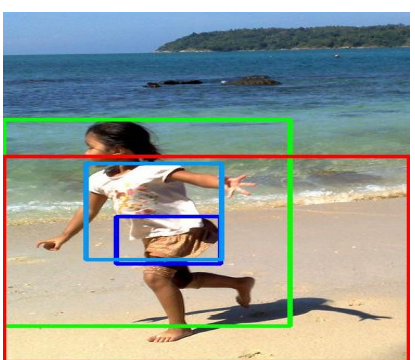

A young girl in shorts and white shirt is running on the beach.

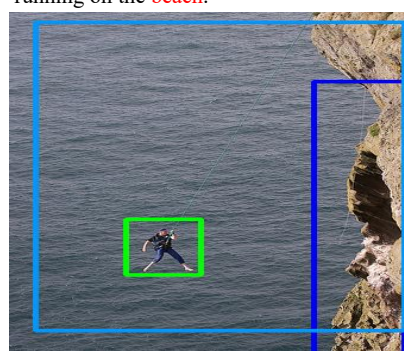

A man is climbing a cliff over the water.

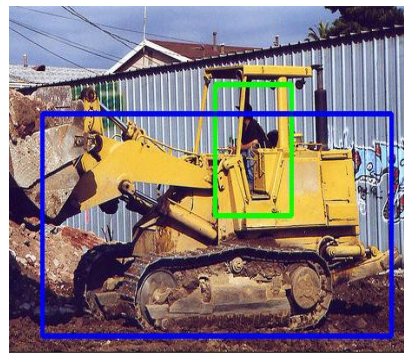

A man is driving a yellow tractor.

Figure 6: Visualization of our generated captions as well as the corresponding grounded regions.

Table 2: Effect of the number of attention branches $K$.

\begin{tabular}{cccccccc}
\hline K & \multicolumn{4}{c}{ Captioning Eval } & \multicolumn{3}{c}{ Grounding Eval } \\
\cline { 2 - 8 } & B@1 & B@4 & M & C & S & $F 1_{\text {all }}$ & $F 1_{\text {loc }}$ \\
\hline 2 & 68.8 & 26.5 & 22.4 & 60.9 & 16.6 & 5.2 & 15.90 \\
3 & 69.3 & 27.0 & 22.4 & 61.6 & 16.7 & 6.78 & 20.10 \\
4 & 69.2 & 27.2 & 22.5 & 62.5 & 16.5 & 7.91 & 21.54 \\
5 & 68.9 & 26.6 & 22.4 & 62.4 & 16.7 & 7.62 & 20.45 \\
\hline
\end{tabular}

13.19 for $F 1_{\text {loc }}$, which is lower than our result as shown in Table 1. One possible reason is that, in our work, we explicitly enforce the attention branches to focus on multiple regions with consistent semantics, which is not guaranteed for multi-head attention.

\subsection{Error Analysis}

In this section, to better understand the grounding performance, we analyzed the grounding accuracy detailly by classifying all the predictions on Flickr30k Entities test set into five categories:
Table 3: Results without region proposal elimination.

\begin{tabular}{cccccccc}
\hline K & \multicolumn{4}{c}{ Captioning Eval } & \multicolumn{3}{c}{ Grounding Eval } \\
\cline { 2 - 8 } & B@1 & B@4 & M & C & S & $F 1_{\text {all }}$ & $F 1_{\text {loc }}$ \\
\hline 2 & 68.9 & 27.0 & 22.4 & 61.1 & 16.5 & 5.48 & 15.80 \\
3 & 68.6 & 27.1 & 22.51 & 61.85 & 16.41 & 5.48 & 16.14 \\
4 & 68.9 & 26.8 & 22.4 & 61.5 & 16.6 & 6.82 & 18.91 \\
5 & 68.9 & 26.8 & 22.3 & 61.4 & 16.3 & 6.27 & 17.36 \\
\hline
\end{tabular}

Mis Cls: Missing Class computes the ratio of noun words that are in the ground truth reference captions but not in the corresponding predicted captions.

Hallu Cls: Hallucinated Class computes the ratio of noun words that are in the predicted captions but not in the corresponding ground truth reference captions.

Corr Grd: Correct Grounding computes the ratio of noun words that are correctly generated and grounded (IOU $>0.5)$.

Part Grd: Partial Grounding computes the ratio of noun words that are correctly generated but partially grounded (the predicted 

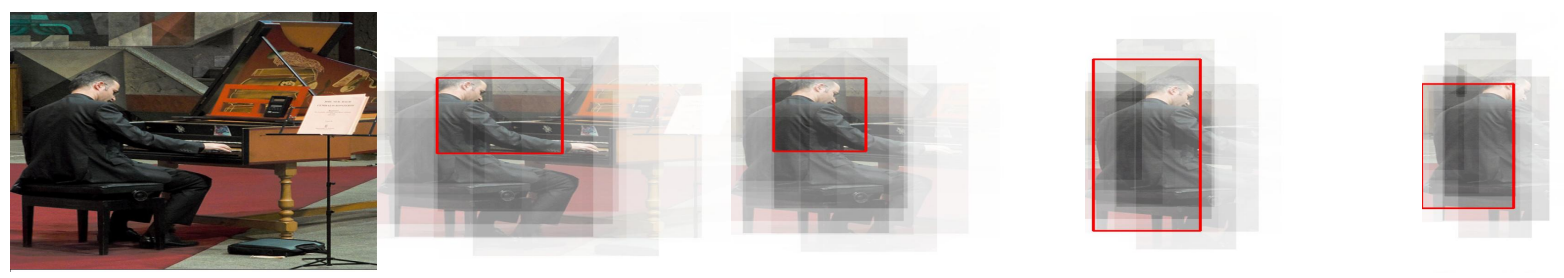

A man in a black suit is sitting at a piano.
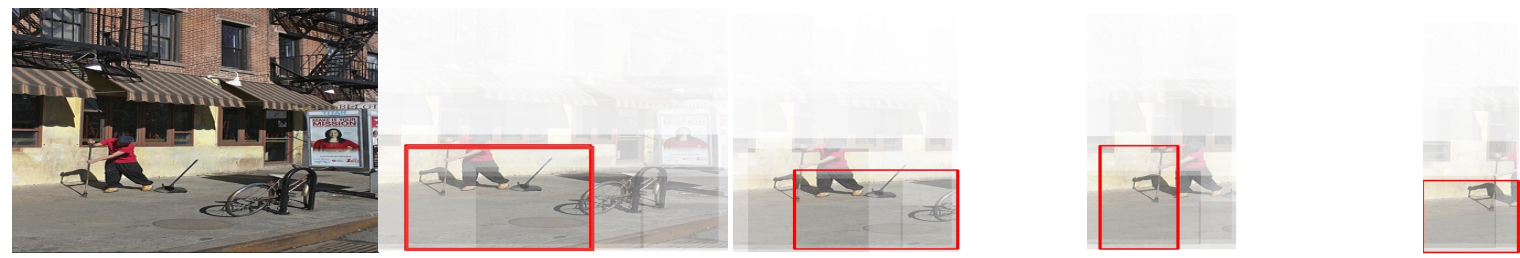

A man in a red shirt and black pants is sweeping the sidewalk.

Figure 7: Visualization of the distributed attention. For a noun word (red) in a generated caption, the corresponding attention weights and the selected regions are shown.

Table 4: Detailed analysis of the grounding accuracy by classifying all the predictions on Flickr30k Entities test set into five types. The ratio of each type is computed.

\begin{tabular}{ccccccc}
\hline & \multicolumn{2}{c}{ Err Cls } & & \multicolumn{3}{c}{ Corr Cls } \\
\cline { 2 - 3 } \cline { 6 - 7 } \cline { 5 - 7 } & Mis Cls & Hallu Cls & & Corr Grd & Part Grd & Ohter Err \\
\hline Baseline & $33.26 \%$ & $\mathbf{4 4 . 9 2 \%}$ & & $7.12 \%$ & $7.94 \%$ & $\mathbf{6 . 7 6 \%}$ \\
Ours & $\mathbf{3 2 . 7 6 \%}$ & $45.32 \%$ & & $\mathbf{1 0 . 9 2 \%}$ & $\mathbf{2 . 0 3 \%}$ & $8.97 \%$ \\
\hline
\end{tabular}

bounding box is contained in the ground truth bounding boxes, but the IOU of them is less than 0.5).

Ohter Err: Other Error Grounding computes the ratio of noun words that are correctly generated but are neither in Part Grd nor in Corr Grd.

As shown in Table 4, the classification error (missing class and hallucinated class) contributes to the most portion of the error when computing the grounding accuracy. One main reason is that, for a given image, there exist various ways for describing its content, and directly comparing the generated captions with the corresponding references may lead to errors in computing the classification accuracy. That's why the metric $F 1_{\text {all }}$, which considers the classification accuracy, is always much lower than $F 1_{l o c}$. And it's also the limitation of current evaluation metrics in this task.

When only considering the predictions whose classification labels are correct, our proposed method achieved much more correctly grounded predictions than that of the baseline method. It further confirmed the importance of solving the partial grounding issue and the superiority of our proposed method.

We also measure the grounding accuracy of the baseline method if regarding all the predictions which are partially grounded as correct predictions to see the upper bound. In this case, the $F 1_{\text {all }}$ and $F 1_{\text {loc }}$ are 10.65 and 30.28 correspondingly. By comparing it with our results $\left(F 1_{\text {all }}: 7.91, F 1_{\text {loc }}: 21.5\right)$, we can see that, there is still a large space for improvement.

\subsection{Limitations}

The main limitation of our proposed method is that the predicted grounding regions might be larger than the target objects as shown in Fig. 8. One important reason might be that the attention weights are calculated based on contextual information from the previous words rather than the one to be generated. Thus, the attention weights predicted by some attention branches might have the 'deviated focus' problem, and fusing the deviated regions together may lead to enlarged grounding regions. One possible solution is to combine our work with other state-of-the-arts (e.g. [26, 31]). As it is not the main focus of our work, we leave it as future work.

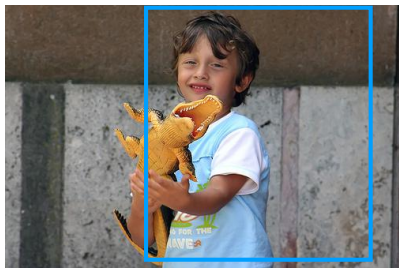

A young boy in a white shirt is holding a toy.

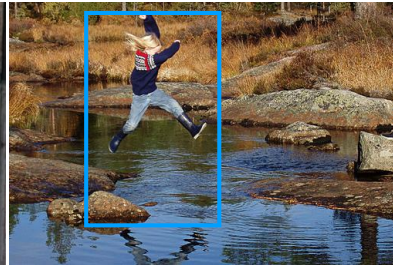

A girl jumping in a stream.
Figure 8: Limitation of our method.

\section{CONCLUSION}

In this paper, we study the problem of generating image captions with accurate grounding regions without any grounding annotations. We unveil the fact that alleviating the partial grounding issue is critical to improve the grounding performance, which has been overlooked in many previous works. To this end, we propose the distributed attention mechanism to enforce the network to aggregate different regions with consistent semantics while generating the words. Extensive experiments have shown the effectiveness of our proposed method. By incorporating our proposed module into the baseline network, we achieved significant improvement on the grounding accuracy compared with the state-of-the-arts. 


\section{REFERENCES}

[1] Peter Anderson, Basura Fernando, Mark Johnson, and Stephen Gould. 2016. Spice: Semantic propositional image caption evaluation. In European conference on computer vision. Springer, 382-398.

[2] Peter Anderson, Xiaodong He, Chris Buehler, Damien Teney, Mark Johnson, Stephen Gould, and Lei Zhang. 2018. Bottom-up and top-down attention for image captioning and visual question answering. In Proceedings of the IEEE conference on computer vision and pattern recognition. 6077-6086.

[3] Samy Bengio, Oriol Vinyals, Navdeep Jaitly, and Noam Shazeer. 2015. Scheduled sampling for sequence prediction with recurrent neural networks. arXiv preprint arXiv:1506.03099 (2015)

[4] Hakan Bilen, Marco Pedersoli, and Tinne Tuytelaars. 2015. Weakly supervised object detection with convex clustering. In Proceedings of the IEEE Conference on Computer Vision and Pattern Recognition. 1081-1089.

[5] Hakan Bilen and Andrea Vedaldi. 2016. Weakly supervised deep detection networks. In Proceedings of the IEEE Conference on Computer Vision and Pattern Recognition. 2846-2854.

[6] Kan Chen, Jiyang Gao, and Ram Nevatia. 2018. Knowledge aided consistency for weakly supervised phrase grounding. In Proceedings of the IEEE Conference on Computer Vision and Pattern Recognition. 4042-4050.

[7] Xinlei Chen, Hao Fang, Tsung-Yi Lin, Ramakrishna Vedantam, Saurabh Gupta, Piotr Dollár, and C Lawrence Zitnick. 2015. Microsoft coco captions: Data collection and evaluation server. arXiv preprint arXiv:1504.00325 (2015).

[8] Junsuk Choe and Hyunjung Shim. 2019. Attention-based dropout layer for weakly supervised object localization. In IEEE CVPR. 2219-2228.

[9] Marcella Cornia, Matteo Stefanini, Lorenzo Baraldi, and Rita Cucchiara. 2020 Meshed-memory transformer for image captioning. In Proceedings of the IEEE/CVF Conference on Computer Vision and Pattern Recognition. 10578-10587.

[10] Pradipto Das, Chenliang Xu, Richard F Doell, and Jason J Corso. 2013. A thousand frames in just a few words: Lingual description of videos through latent topics and sparse object stitching. In Proceedings of the IEEE conference on computer vision and pattern recognition. 2634-2641.

[11] Michael Denkowski and Alon Lavie. 2014. Meteor universal: Language specific translation evaluation for any target language. In Proceedings of the ninth workshop on statistical machine translation. 376-380.

[12] Pelin Dogan, Leonid Sigal, and Markus Gross. 2019. Neural sequential phrase grounding (seqground). In Proceedings of the IEEE/CVF Conference on Computer Vision and Pattern Recognition. 4175-4184.

[13] Wei Gao, Fang Wan, Xingjia Pan, Zhiliang Peng, Qi Tian, Zhenjun Han, Bolei Zhou, and Qixiang Ye. 2021. TS-CAM: Token Semantic Coupled Attention Map for Weakly Supervised Object Localization. arXiv preprint arXiv:2103.14862 (2021).

[14] Ramazan Gokberk Cinbis, Jakob Verbeek, and Cordelia Schmid. 2014. Multi-fold mil training for weakly supervised object localization. In Proceedings of the IEEE conference on computer vision and pattern recognition. 2409-2416.

[15] Longteng Guo, Jing Liu, Xinxin Zhu, Peng Yao, Shichen Lu, and Hanqing Lu 2020. Normalized and Geometry-Aware Self-Attention Network for Image Captioning. In Proceedings of the IEEE/CVF Conference on Computer Vision and Pattern Recognition. 10327-10336.

[16] Tanmay Gupta, Arash Vahdat, Gal Chechik, Xiaodong Yang, Jan Kautz, and Derek Hoiem. 2020. Contrastive learning for weakly supervised phrase grounding. arXiv preprint arXiv:2006.09920 (2020).

[17] Kaiming He, Xiangyu Zhang, Shaoqing Ren, and Jian Sun. 2016. Deep residual learning for image recognition. In Proceedings of the IEEE conference on computer vision and pattern recognition. $770-778$.

[18] Lun Huang, Wenmin Wang, Jie Chen, and Xiao-Yong Wei. 2019. Attention on attention for image captioning. In Proceedings of the IEEE/CVF International Conference on Computer Vision. 4634-4643.

[19] Vadim Kantorov, Maxime Oquab, Minsu Cho, and Ivan Laptev. 2016. Contextlocnet: Context-aware deep network models for weakly supervised localization. In European Conference on Computer Vision. Springer, 350-365.

[20] Andrej Karpathy and Li Fei-Fei. 2015. Deep visual-semantic alignments for generating image descriptions. In Proceedings of the IEEE conference on computer vision and pattern recognition. 3128-3137.

[21] Diederik P Kingma and Jimmy Ba. 2014. Adam: A method for stochastic optimization. arXiv preprint arXiv:1412.6980 (2014).

[22] Ranjay Krishna, Yuke Zhu, Oliver Groth, Justin Johnson, Kenji Hata, Joshua Kravitz, Stephanie Chen, Yannis Kalantidis, Li-Jia Li, David A Shamma, et al. 2017. Visual genome: Connecting language and vision using crowdsourced dense image annotations. International journal of computer vision 123, 1 (2017), 32-73.

[23] Girish Kulkarni, Visruth Premraj, Vicente Ordonez, Sagnik Dhar, Siming Li, Yejin Choi, Alexander C Berg, and Tamara L Berg. 2013. Babytalk: Understanding and generating simple image descriptions. IEEE Transactions on Pattern Analysis and Machine Intelligence 35, 12 (2013), 2891-2903.

[24] Kuang-Huei Lee, Xi Chen, Gang Hua, Houdong Hu, and Xiaodong He. 2018. Stacked cross attention for image-text matching. In Proceedings of the European Conference on Computer Vision (ECCV). 201-216.

[25] Chenxi Liu, Junhua Mao, Fei Sha, and Alan Yuille. 2017. Attention correctness in neural image captioning. In Proceedings of the AAAI Conference on Artificial
Intelligence, Vol. 31

[26] Fenglin Liu, Xuancheng Ren, Xian Wu, Shen Ge, Wei Fan, Yuexian Zou, and Xu Sun. 2020. Prophet Attention: Predicting Attention with Future Attention. Advances in Neural Information Processing Systems 33 (2020).

[27] Xuejing Liu, Liang Li, Shuhui Wang, Zheng-Jun Zha, Li Su, and Qingming Huang. 2019. Knowledge-guided pairwise reconstruction network for weakly supervised referring expression grounding. In Proceedings of the 27th ACM International Conference on Multimedia. 539-547.

[28] Yongfei Liu, Bo Wan, Lin Ma, and Xuming He. 2021. Relation-aware Instance Refinement for Weakly Supervised Visual Grounding. arXiv preprint

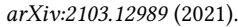

[29] Yongfei Liu, Bo Wan, Xiaodan Zhu, and Xuming He. 2020. Learning cross-modal context graph for visual grounding. In Proceedings of the AAAI Conference on Artificial Intelligence, Vol. 34. 11645-11652.

[30] Jiasen Lu, Caiming Xiong, Devi Parikh, and Richard Socher. 2017. Knowing when to look: Adaptive attention via a visual sentinel for image captioning. In Proceedings of the IEEE conference on computer vision and pattern recognition. 375-383.

[31] Chih-Yao Ma, Yannis Kalantidis, Ghassan AlRegib, Peter Vajda, Marcus Rohrbach, and Zsolt Kira. 2020. Learning to generate grounded visual captions without localization supervision. In Proceedings of the European Conference on Computer Vision (ECCV), Vol. 2. Springer.

[32] Margaret Mitchell, Jesse Dodge, Amit Goyal, Kota Yamaguchi, Karl Stratos, Xufeng Han, Alyssa Mensch, Alexander Berg, Tamara Berg, and Hal Daumé III. 2012. Midge: Generating image descriptions from computer vision detections. In Proceedings of the 13th Conference of the European Chapter of the Association for Computational Linguistics. 747-756.

[33] Xingjia Pan, Yingguo Gao, Zhiwen Lin, Fan Tang, Weiming Dong, Haolei Yuan, Feiyue Huang, and Changsheng Xu. 2021. Unveiling the Potential of Structure-Preserving for Weakly Supervised Object Localization. arXiv preprint arXiv:2103.04523 (2021)

[34] Kishore Papineni, Salim Roukos, Todd Ward, and Wei-Jing Zhu. 2002. Bleu: a method for automatic evaluation of machine translation. In Proceedings of the 40th annual meeting of the Association for Computational Linguistics. 311-318.

[35] Bryan A Plummer, Paige Kordas, M Hadi Kiapour, Shuai Zheng, Robinson Piramuthu, and Svetlana Lazebnik. 2018. Conditional image-text embedding networks. In Proceedings of the European Conference on Computer Vision (ECCV). 249-264.

[36] Bryan A Plummer, Liwei Wang, Chris M Cervantes, Juan C Caicedo, Julia Hockenmaier, and Svetlana Lazebnik. 2015. Flickr30k entities: Collecting region-tophrase correspondences for richer image-to-sentence models. In Proceedings of the IEEE international conference on computer vision. 2641-2649.

[37] Shaoqing Ren, Kaiming He, Ross Girshick, and Jian Sun. 2015. Faster r-cnn: Towards real-time object detection with region proposal networks. arXiv preprint arXiv:1506.01497 (2015).

[38] Steven J Rennie, Etienne Marcheret, Youssef Mroueh, Jerret Ross, and Vaibhava Goel. 2017. Self-critical sequence training for image captioning. In Proceedings of the IEEE Conference on Computer Vision and Pattern Recognition. 7008-7024.

[39] Anna Rohrbach, Marcus Rohrbach, Ronghang Hu, Trevor Darrell, and Bernt Schiele. 2016. Grounding of textual phrases in images by reconstruction. In European Conference on Computer Vision. Springer, 817-834.

[40] Yunhang Shen, Rongrong Ji, Kuiyuan Yang, Cheng Deng, and Changhu Wang. 2019. Category-aware spatial constraint for weakly supervised detection. IEEE Transactions on Image Processing 29 (2019), 843-858.

[41] Krishna Kumar Singh and Yong Jae Lee. 2017. Hide-and-seek: Forcing a network to be meticulous for weakly-supervised object and action localization. In IEEE ICCV. 3544-3553.

[42] Hyun Oh Song, Ross Girshick, Stefanie Jegelka, Julien Mairal, Zaid Harchaoui, and Trevor Darrell. 2014. On learning to localize objects with minimal supervision. In International Conference on Machine Learning. PMLR, 1611-1619.

[43] Eu Wern Teh, Mrigank Rochan, and Yang Wang. 2016. Attention Networks for Weakly Supervised Object Localization.. In BMVC. 1-11.

[44] Ashish Vaswani, Noam Shazeer, Niki Parmar, Jakob Uszkoreit, Llion Jones, Aidan N Gomez, Lukasz Kaiser, and Illia Polosukhin. 2017. Attention is all you need. arXiv preprint arXiv:1706.03762 (2017).

[45] Ramakrishna Vedantam, C Lawrence Zitnick, and Devi Parikh. 2015. Cider: Consensus-based image description evaluation. In Proceedings of the IEEE conference on computer vision and pattern recognition. 4566-4575.

[46] Oriol Vinyals, Alexander Toshev, Samy Bengio, and Dumitru Erhan. 2015. Show and tell: A neural image caption generator. In Proceedings of the IEEE conference on computer vision and pattern recognition. 3156-3164.

[47] Liwei Wang, Jing Huang, Yin Li, Kun Xu, Zhengyuan Yang, and Dong Yu. 2020. Improving Weakly Supervised Visual Grounding by Contrastive Knowledge Distillation. arXiv preprint arXiv:2007.01951 (2020).

[48] Yunchao Wei, Zhiqiang Shen, Bowen Cheng, Honghui Shi, Jinjun Xiong, Jiashi Feng, and Thomas Huang. 2018. Ts2c: Tight box mining with surrounding segmentation context for weakly supervised object detection. In Proceedings of the European Conference on Computer Vision (ECCV). 434-450. 
[49] Xu Yang, Kaihua Tang, Hanwang Zhang, and Jianfei Cai. 2019. Auto-encoding scene graphs for image captioning. In Proceedings of the IEEE/CVF Conference on Computer Vision and Pattern Recognition. 10685-10694.

[50] Quanzeng You, Hailin Jin, Zhaowen Wang, Chen Fang, and Jiebo Luo. 2016. Image captioning with semantic attention. In Proceedings of the IEEE conference on computer vision and pattern recognition. 4651-4659.

[51] Licheng Yu, Zhe Lin, Xiaohui Shen, Jimei Yang, Xin Lu, Mohit Bansal, and Tamara L Berg. 2018. Mattnet: Modular attention network for referring expression comprehension. In Proceedings of the IEEE Conference on Computer Vision and Pattern Recognition. 1307-1315.

[52] Youngjae Yu, Jongwook Choi, Yeonhwa Kim, Kyung Yoo, Sang-Hun Lee, and Gunhee Kim. 2017. Supervising neural attention models for video captioning by human gaze data. In Proceedings of the IEEE Conference on Computer Vision and Pattern Recognition. 490-498.

[53] Sangdoo Yun, Dongyoon Han, Seong Joon Oh, Sanghyuk Chun, Junsuk Choe, and Youngjoon Yoo. 2019. Cutmix: Regularization strategy to train strong classifiers with localizable features. In Proceedings of the IEEE International Conference on Computer Vision. 6023-6032.

[54] Wenqiao Zhang, Xin Eric Wang, Siliang Tang, Haizhou Shi, Haochen Shi, Jun Xiao, Yueting Zhuang, and William Yang Wang. 2020. Relational Graph Learning for Grounded Video Description Generation. In Proceedings of the 28th ACM International Conference on Multimedia. 3807-3828.

[55] Xiaolin Zhang, Yunchao Wei, Jiashi Feng, Yi Yang, and Thomas S Huang. 2018. Adversarial complementary learning for weakly supervised object localization. In IEEE CVPR. 1325-1334.

[56] Xiaolin Zhang, Yunchao Wei, Guoliang Kang, Yi Yang, and Thomas Huang. 2018. Self-produced guidance for weakly-supervised object localization. In ECCV. 597613.

[57] Xiaolin Zhang, Yunchao Wei, and Yi Yang. 2020. Inter-image communication for weakly supervised localization. In ECCV, Vol. 12364. 271-287.

[58] Bolei Zhou, Aditya Khosla, Agata Lapedriza, Aude Oliva, and Antonio Torralba. 2016. Learning deep features for discriminative localization. In Proceedings of the IEEE conference on computer vision and pattern recognition. 2921-2929.

[59] Luowei Zhou, Yannis Kalantidis, Xinlei Chen, Jason J Corso, and Marcus Rohrbach. 2019. Grounded video description. In Proceedings of the IEEE/CVF Conference on Computer Vision and Pattern Recognition. 6578-6587.

[60] Yuanen Zhou, Meng Wang, Daqing Liu, Zhenzhen Hu, and Hanwang Zhang. 2020. More grounded image captioning by distilling image-text matching model. In Proceedings of the IEEE/CVF Conference on Computer Vision and Pattern Recognition. $4777-4786$. 